Check for updates

Cite this: RSC Adv., 2018, 8, 6789

Received 15th December 2017 Accepted 30th January 2018

DOI: 10.1039/c7ra13360j

rsc.li/rsc-advances

\section{Triple network hydrogels (TN gels) prepared by a one-pot, two-step method with high mechanical properties}

\author{
Xiangong Wang, Fang Zhao, Bo Pang, Xuping Qin* and Shengyu Feng (D)* \\ In this work, poly(vinyl alcohol) (PVA) was incorporated into the networks of polyacrylamide/polyacrylic acid \\ (PAM/PAA) to prepare novel PAM/PAA/PVA Triple-network (TN) hydrogels by an in situ polymerization and \\ repeated freezing-thawing ( $\mathrm{F}-\mathrm{T}$ ) process. The TN hydrogels have not only high mechanical strength, but \\ also a moderate swelling ability by varying the weight ratio of calcium chloride $\left(\mathrm{CaCl}_{2}\right)$ and PVA and free \\ shaping. The compressive stress of the as-prepared hydrogels could reach $11 \mathrm{MPa}$, and the highest \\ stretching stress could reach 0.8 MPa. Upon mechanical loading, the coordination network between PAA \\ and $\mathrm{CaCl}_{2}$ served as sacrificial bonds to efficiently dissipate energy. However, they can reform when the \\ mechanical load is released, resulting from the fast coordination between PAA and $\mathrm{Ca}^{2+}$. Therefore, TN \\ hydrogels have potential application in biomaterials.
}

\section{Introduction}

Hydrogels are hydrophilic three-dimensional polymers that have remarkable capacities to hold water and have achieved much progress in sensing, ${ }^{1,2}$ drug delivery, ${ }^{3}$ actuation, ${ }^{4}$ tissue engineering and so on..$^{5-8}$ Both synthetic and natural polymers can be used for the production of hydrogels, ${ }^{9-12}$ achieved by methods such as physical crosslinking, ${ }^{13}$ chemical gelation or self-assembly. ${ }^{14-16}$

The combination of covalent and physical bonds has been proven as an ideal method to produce a hydrogel with high strength, elongation, and excellent recovery properties. ${ }^{17}$ Cations can form coordination bonds with the appropriate ligands and, in most cases, dissociate and associate reversibly and rapidly. ${ }^{18-20}$ This has led to their use as physical crosslink points for preparing high strength hydrogels because of the ease of energy dissipation and recovery of mechanical properties in a short amount of time..$^{21,22}$

Articular cartilage can reach $60 \%$ of compressibility strain, and possesses the excellent mechanical properties of quick stress relaxation, large energy dissipation, strain stiffening, and fast recovery of the mechanical properties after the release of load. ${ }^{23}$ The cartilage has a water content of $70-80 \%$ from its extracellular matrix composition..$^{24,25}$ Inspired by the mechanical properties of articular cartilage, many tough hydrogels have been designed with double-network hydrogels (DN gels). ${ }^{26,27}$ For instance, Suo et al. prepared a new type of DN hydrogel through

Key Laboratory of Special Functional Aggregated Materials, Key Laboratory of Colloid and Interface Chemistry, Ministry of Education, School of Chemistry and Chemical Engineering, Shandong University, Jinan 250100, P. R. China. E-mail: fsy@sdu.edu. cn; qinxvping@sdu.edu.cn combining the networks of PAM and alginate (ionic), which is notch-insensitive, very tough and stretchable up to 20 times the initial length, but still soft, with a tensile strength of only $\approx 160 \mathrm{kPa} .^{28} \mathrm{Yu}$ et al. designed a stretchable and tough hydrogel cross-linked between $\mathrm{Ca}^{2+}$ and graphene oxide. But its tensile stress is also only about $150 \mathrm{kPa}$.

PVA is a water soluble synthetic polymer of great interest because of its many desirable characteristics, such as its viscoelastic nature, high water content, and biocompatibility. Therefore, it is advantageous to use it as a biomaterial for fabricating medical devices to be used as synthetic articular cartilage..$^{29} \mathrm{PVA}$ can be a semi-crystalline structure, formed through intramolecular hydrogen bonding, resulting from the possession of a large number of hydroxyl side groups. PVA can be made into hydrogels through chemical or physical cross-linking, such as glutaraldehyde acting as a functional cross-linking agents, ${ }^{30}$ or ionizing radiation. Physically cross-linked PVA-based hydrogels in water can be prepared by the F-T process, in which, the concentrated PVA aqueous solution induces crystallization, leading to the formation of a network structure..$^{31}$ Alternatively, PVA physical hydrogels have drawn even greater research attention, in which, this method addresses toxicity issues because it does not result in the reagent residuals and chemical toxicity; consequently, no toxicity agents leaching from the gel. Furthermore, these physically cross-linked hydrogels, which are inter-connected by hydrogen bonding, exhibit more porous, spongy, rubbery and higher elastic properties than PVA hydrogels prepared by other methods. ${ }^{32}$ Currently, these gel matrices have been widely implemented in biotechnology fields. Crystallites serve as physical cross-links that are both reversible and stable, but, unfortunately, PVA hydrogels synthesized using this method are brittle. ${ }^{33}$ 
Most hydrogels have low stiffness $(10 \mathrm{kPa})$, strength $(100 \mathrm{kPa})$ and toughness $\left(10 \mathrm{~J} \mathrm{~m}^{-2}\right) \cdot \cdot^{34,35}$ Despite recent progress, it is imperative to develop effective methods of preparing novel hydrogels with excellent comprehensive performance of in terms of mechanical properties, ${ }^{36,37}$ the ability to control waterabsorption, and recovery properties in the as-prepared state, as well as in the swelling equilibrium states.

In this work, we have prepared hydrogels with a low covalently cross-linked PAM plus PAA network, a physically crosslinked PVA network through crystallizing by the F-T process, and an ionically cross-linked network between PAA and $\mathrm{Ca}^{2+}$. In this gels, acrylamide (AM) and acrylic acid (AA) were made for the first network cross-linking, with micro-scale a chemical cross-linker, through an in situ polymerization. Meanwhile, the chains of PVA interpenetrated with network of PAM, PAA and the F-T process, resulting in crystallites, which acted as junction points in the hydrogel. At the same time, the ionic cross-linking between PAA and $\mathrm{Ca}^{2+}$ made another interpenetration with PVA, in which the third network was created (Scheme 1). All PAM, PAA chains and PVA chains form a continuous network, which closely correlates with the mechanical properties of the TN gels. The as-prepared hydrogels coexisted physical network and covalent network. Ionic bonds and crystallites have been used as the sacrificial network, which greatly improved the recovery of the mechanical stability. Based on the coexisting high density physical bonds, stress could be greatly dissipated by unzipping physical bonds during the deformation process.

The Triple network (TN) has a synergetic effect, which can dramatically increase the mechanical properties. Because the robust hydrogels were synthesized via a one-pot, two-step procedure, the hydrogels can be prepared in different desired shapes controlled by the shapes of the mould.

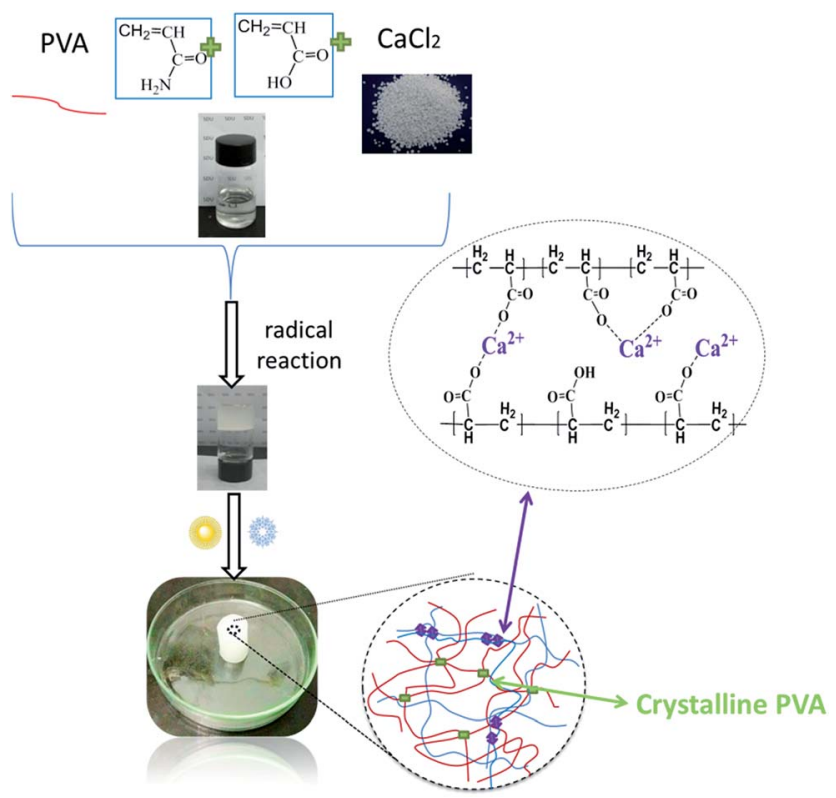

Scheme 1 Preparation of TN gels by using one-pot, two step method.

\section{Results and discussion}

\section{Mechanical properties}

The typical stress-strain curves of the prepared hydrogels are shown in Fig. 1. From Fig. 1a, with a fixed weight ratio of AM/AA at 2 and the content of $\mathrm{CaCl}_{2}$ at $4.2 \%(\mathrm{w} / \mathrm{v})$, the tensile stress and toughness gradually increase with the increasing content of PVA, while the elongation decreased. The tensile strength of the 2-P0C4.17 TN gel is only $\approx 61 \mathrm{kPa}$ at a strain of $1045 \%$. In contrast, the stress of the 2-P8.3-4.17 TN gel can reach $0.804 \mathrm{MPa}$, which is 13 times higher than that of the 2-P0-C4.17 TN gels. The corresponding tensile strain is $365 \%$, which is much less than that of the 2-P8.3-4.17 TN gels, and the toughness ${ }^{38}$ is $1.505 \mathrm{M} \mathrm{J}^{-3}$. The results indicate that the introduction of PVA can significantly improve the mechanical strength, excluding the tensile strain. This occurs from the physical cross-linked density of the as-prepared hydrogel, which is increased through the F-T process and the molecular entanglement between PAM, PAA and PVA, which was strengthened by introducing more PVA. Futheremore, the tensile strain decreased with increasing physical cross-linked density.

The compressive stress-strain curves of the hydrogels are shown in Fig. 1b. The as-prepared hydrogels could be compressed to a strain of $90 \%$, and the stress at this strain was used to evaluate the compressive strength of the as-prepared hydrogels. The curves of the as-prepared hydrogels, which went through the F-T process for a total of three cycle showed that the compressive stress decreased with increasing content of PVA. At this condition, the ionic coordination bonds of the hydrogels play an important role in maintaining the excellent compressive performance.

Another series of experiments' fixed the content of PVA and $\mathrm{CaCl}_{2}$ but with a changing weight ratio of AM/AA (Fig. 2). When the weight ratio of AM/AA increased from 0.2 to 1 , the tensile strength increased considerably, from $64.3 \mathrm{kPa}$ to $0.572 \mathrm{MPa}$, and the corresponding ruptured strain increased from $417 \%$ to $612 \%$ (Fig. 2a). For the different weight ratio of AM/AA, $0.572 \mathrm{MPa}$ was the highest tensile strength. When the weight ratio of $\mathrm{AM} / \mathrm{AA}$ increased from 1 to 5 , the tensile strength decreased considerably from $0.572 \mathrm{MPa}$ to $0.263 \mathrm{MPa}$ and the corresponding ruptured strains decreased from $612 \%$ to $491 \%$. The toughness increased dramatically, from near $0.6 \mathrm{M} \mathrm{J} \mathrm{m}^{-3}$ to 1.7 $\mathrm{M} \mathrm{J} \mathrm{m}^{-3}$, with the decreasing weight ratio of AM/AA (Fig. 2c). The results indicated that the mechanical strength of the hydrogel has a strong dependence on the weight ratios of AM/AA. Mechanical strength can be changed by regulating the content of AA, influencing the coordination interaction.

The compressive strain-stress curves of the as-prepared hydrogels are shown in Fig. 2b. The compressive stress increased with the decreasing weight ratio of $\mathrm{AM} / \mathrm{AA}$, in which the rules of change differ from those of the stretching stress. The results indicated that the compressive stress was enhanced with increasing AA content and excess $\mathrm{Ca}^{2+}$ cross-linking with PAA, resulting in increased ion coordination bonds, and improving the compressive strength resulting from the physical cross-linked density.

Another series of experiments were conducted, in which the content of PVA and ratio of AM/AA were fixed, with only the 

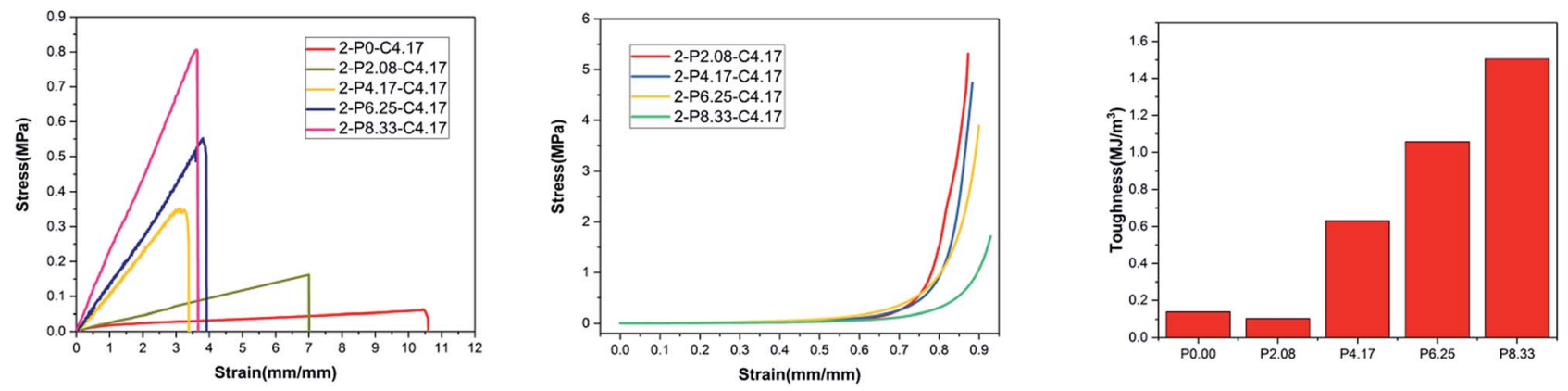

Fig. 1 Stress-strain curves (a), compressive stress-strain curves (b) and toughness (c) of the hydrogels at various content of PVA.

content of $\mathrm{CaCl}_{2}$ varied (Fig. 3). The breaking strain changed slightly with the increasing content of $\mathrm{CaCl}_{2}$, and the stretching stress of the 2-P4.17-C0.92 TN gels increased from 0.30 MPa to 0.35 MPa of the 2-P4.17-C4.17 TN gels. Therefore, the results suggest that the influence of $\mathrm{CaCl}_{2}$ on the stress and strain was getting slightly with the increasing content of $\mathrm{CaCl}_{2}$. Excess $\mathrm{CaCl}_{2}$ limited improvement of the stress and strain of the asprepared hydrogels, such that superfluous $\mathrm{Ca}^{2+}$ diffused into the hydrogels, leading to enlargement of the ratio of monodentate/bi-edentates. The toughness (Fig. 3c) showed that different content of $\mathrm{CaCl}_{2}$ had different influences on the stress and strain. The results of the compressive strain-stress analysis of the as-prepared hydrogels are shown in Fig. 3b; compressive stress increased with the decreasing content of $\mathrm{CaCl}_{2}$.

All samples could sustain knotting (Fig. 4a), torsion (Fig. 4b), elongation (Fig. 4c-d), compression (Fig. 4e-g). Moreover, they had the ability to recover to the initial state when the load was released. The knotting behaviour of the TN gels indicated that they were highly elastic and stretchable. The 2-P4.17-C0.21 TN gels with a diameter of $8 \mathrm{~mm}$, could lift up a steel block of $\approx 1.5 \mathrm{~kg}$ (Fig. $4 \mathrm{~h}$ and i) which directly indicates that the hydrogels have high mechanical strength. For the sake of comparison, the 0.2-P4.17-C4.17 TN gels went freezing-thawing for a single cycle and three cycles to reveal if the number of $\mathrm{F}-\mathrm{T}$ cycle has an important influence on the compressive stress of the as-prepared hydrogels. The results (Fig. 5a) showed that the compressive stress could increase 10 times with three cycles of the $\mathrm{F}-\mathrm{T}$ process, in comparison to a single cycle of the hydrogel. Therefore, the number of $\mathrm{F}-\mathrm{T}$ cycles can improve the compressive stress of the hydrogels, increasing the physical cross-linked density with the increasing number of F-T cycles.

The loading-unloading tests were measured to evaluate the energy dissipation of the TN gels. Successive loading-unloading cycles were applied to the 2-P4.17-C0.21 TN gels, with testing aimed to make the hydrogels experience enough fatigue. Five cycles of $275 \%$ stretching loading-unloading results are shown in Fig. $5 \mathrm{~b}$ and 3 cycles of $80 \%$ compressing loading-unloading are shown in Fig. 5c. For the as-prepared hydrogels, the residual strain is almost $0 \%$, and a large obvious hysteresis loop can be observed in the first loading-unloading. During the immediate second to fifth cycles of loading-unloading experiments, the residual strain is the same to the first loading-unloading test, with almost no hysteresis observed. This indicated that the TN structure was only changed by the first - loading process, owing to the hydrogel' robustness and ability to undergo multiple loading-unloading cyclic stretching experiments without damage. Meanwhile, the almost unchanged hysteresis loops also demonstrated that during the multiple loading-unloading cyclic stretching experiments, stress was well dissipated by the physical coactions of the $\mathrm{Ca}^{2+}$ and PAA chain unzipping process and the damage to PVA crystallites, while chemical cross-linked networks of the hydrogels remained intact. From Fig. 5c, the 2- a

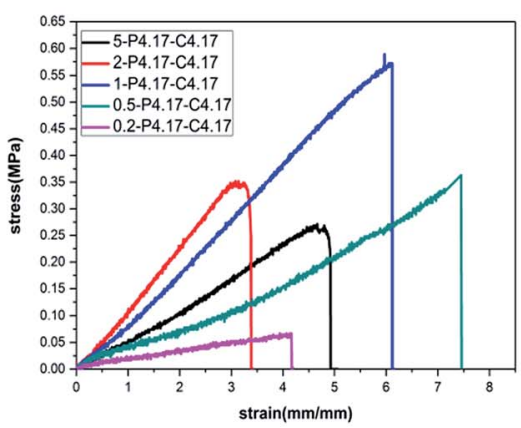

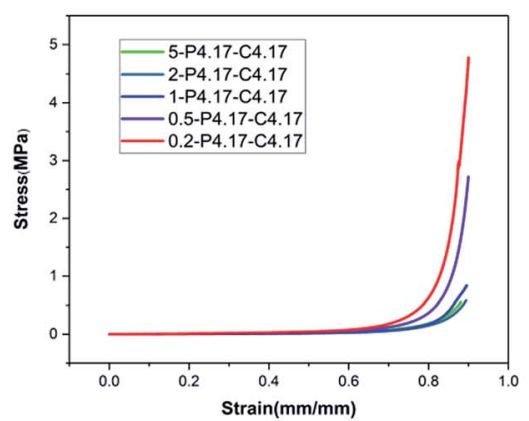

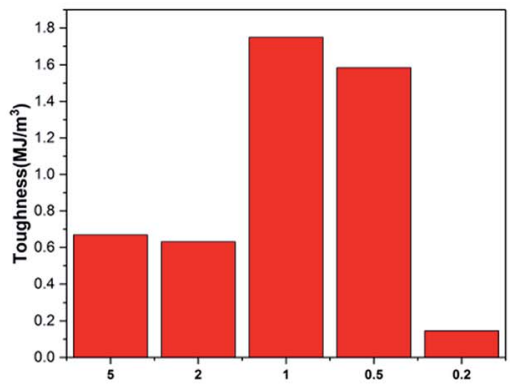

Fig. 2 Stress-strain curves (a), compressive stress-strain curves (b) and toughness (c) of the hydrogels with changing the weight ratio of AM/AA. 

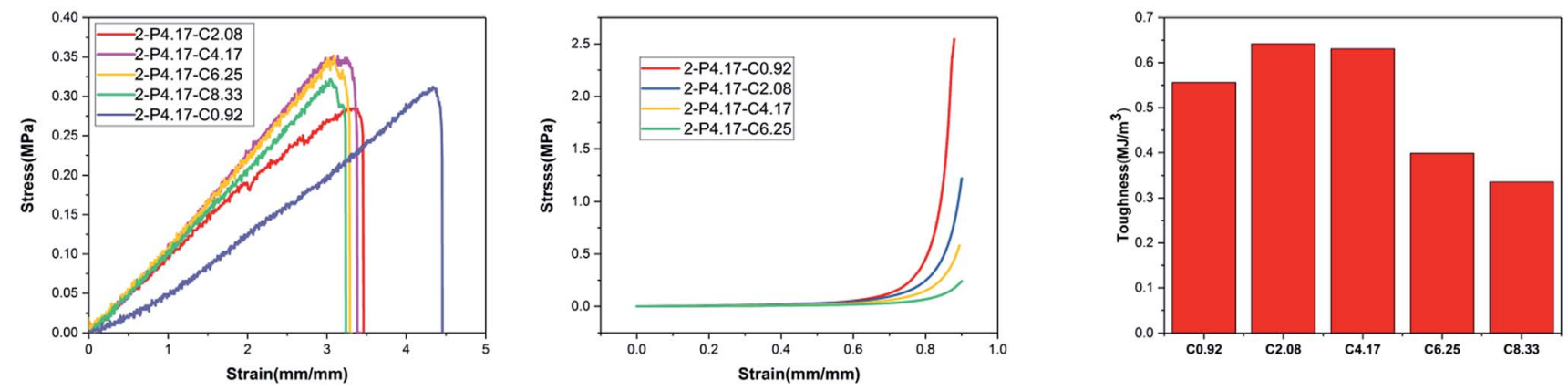

Fig. 3 Stress-strain curves (a), compressive stress-strain curves (b) and toughness (c) of the hydrogels at various content of $\mathrm{CaCl}_{2}$.

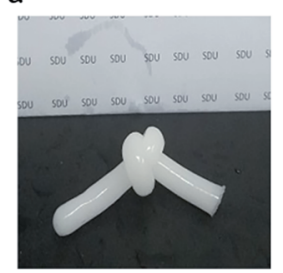

b
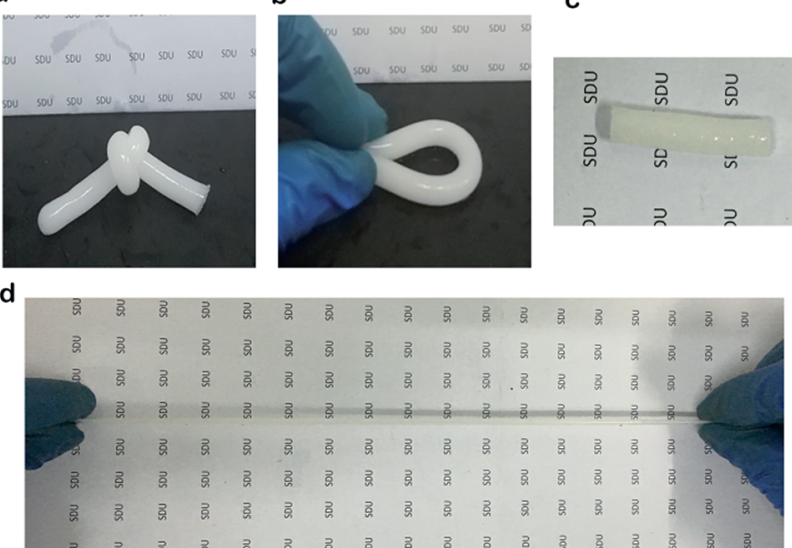

e

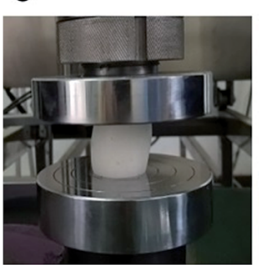

h

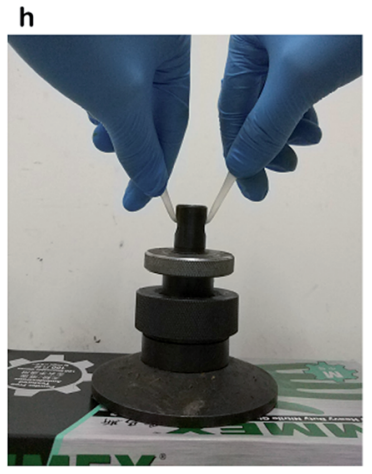

i

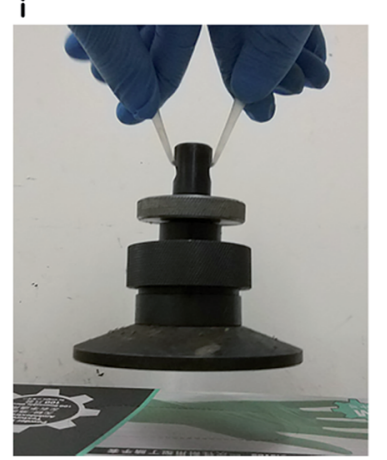

Fig. 4 Compressive and tensile properties of TN gels. (a) Knotting. (b) Torsion. (c, d) Elongation. (e-g) Compression. (h, i) The 2-P4.17-C0.21 $\mathrm{TN}$ gels with a diameter of $8 \mathrm{~mm}$ lift up steel block of $\approx 1.5 \mathrm{~kg}$ in weight.

P4.17-C0.21 TN gels expressed a large hysteresis loop in the first loading-unloading cycle, while in the second and third compression loading-unloading cycles, no hysteresis can be observed, with the last 2 cycles almost overlapping with the first unloading curve. The hydrogels can almost fully recover their mechanical properties within a few seconds. This finding indicates that the $\mathrm{TN}$ structure was changed at the first compression loading process and that the hydrogel only dissipated energy effectively at the first compression cycle. The pure PVA hydrogels produced by the $\mathrm{F}-\mathrm{T}$ process are unrecoverable; the physical bonds result from the fast self-assembly speed and high self-assembly fidelity between PAA and $\mathrm{Ca}^{2+}$. The almost unchanged hysteresis loops indicated that, during the multiple loading-unloading cyclic compression experiments, stress was well dissipated by the physical coaction during the $\mathrm{Ca}^{2+}$ and PAA unzipping process and damage to the PVA crystallites.

Fig. 6a shows the loading-unloading curves at different strains for the 2-P4.17-C0.21 TN gels. Apparent hysteresis loops are observed on the loading-unloading curves, indicating that the 2P4.17-C0.21 TN gels dissipate energy effectively. The data are in Fig. 6b, which clearly shows that the as-prepared hydrogel could dissipate energy as much as $0.086 \mathrm{M} \mathrm{J} \mathrm{m}^{-3}$ at a strain of $325 \%$. Furthermore, there was no hysteresis loop for the sample hydrogel at a strain of $100 \%$, indicating that the hydrogel could not dissipate energy and that it exhibited typical rubber elastic behaviour.

The compressive stress-strain curves of the as-prepared hydrogel and hydrogel with $91.1 \%$ water content are shown in Fig. 7a. The results of compressive experiments show that the hydrogel with $91.1 \%$ water content began its destruction at a stress of 4.5 MPa, without reaching a strain of $90 \%$. Alternatively, the asprepared hydrogel could reach a stress of $5 \mathrm{MPa}$ at a strain of $90 \%$ without destruction. However, the compressive stress of the hydrogel with $91.1 \%$ water content was higher than the asprepared hydrogel at the same strain (before an $80 \%$ strain). Therefore, the results indicate that hydrogels with high water content have excellent performance during the compressing process before reaching an $80 \%$ strain, and the highest compressive stress of the as-prepared hydrogel reached 11 MPa (Fig. 7b).

\section{SEM results}

Fig. 8 depicts the SEM image of cross-section morphologies of the sample. According to Fig. 8a and b, the PAM hydrogels exhibited a closely packed honeycomb-like structure. The crosssection morphology of the 2-P4.17-C0.21 TN gels (freeze-dried) 


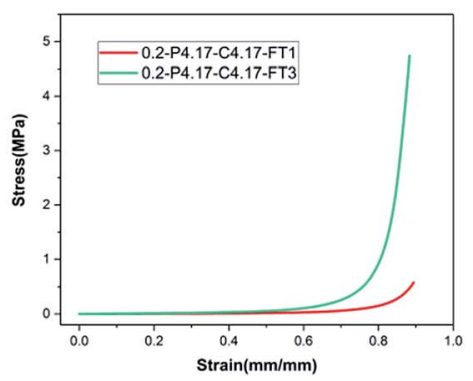

b

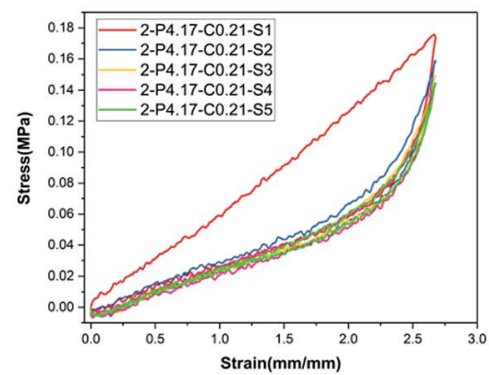

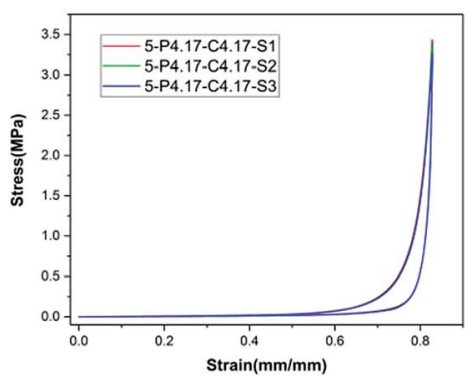

Fig. 5 (a) Compressive stress-strain curves of the 0.2-P4.17-C4.17 TN gels with one cycle or three cycles of the F-T process. (b) Five successive loading-unloading cycles of the as-prepared samples. (c) Compressive stress-strain curves at a strain of $80 \%$ for three successive loadingunloading cycles.

a

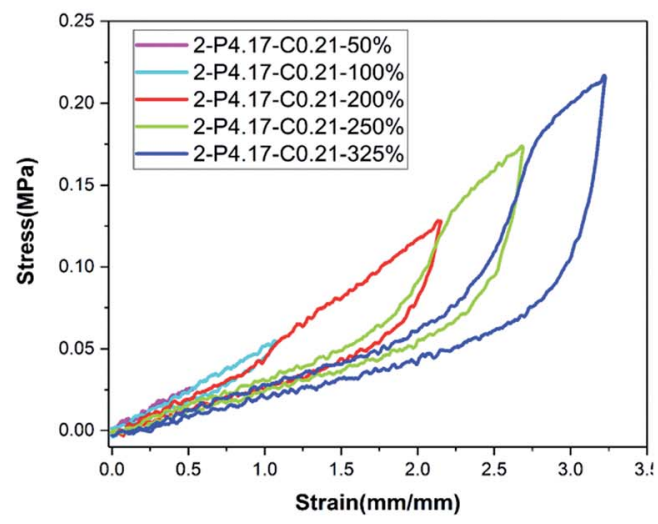

b

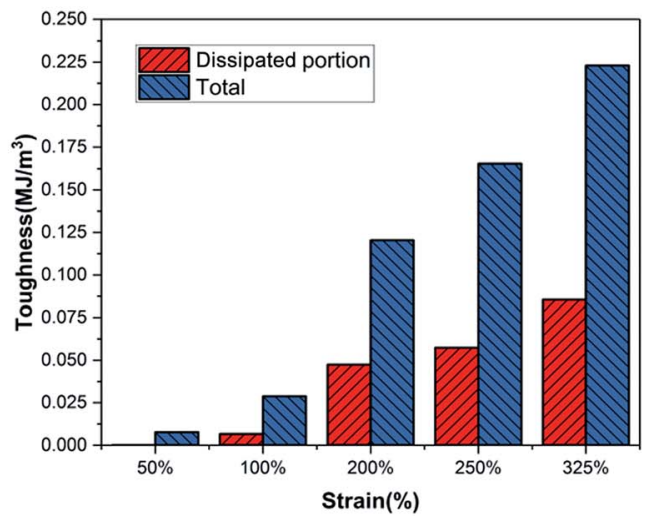

Fig. 6 (a) Loading-unloading tests of the 2-P4.17-C0.21 TN gels under different strain (50\%, 100\%, 200\%, 250\%, 325\%), (b) the calculated total toughness and the dissipated toughness of the 2-P4.17-C0.21 TN gels during the loading-unloading tests under different strains.

is shown in Fig. 8c and d. From Fig. 8c, numerous spines and a few pores were present. And the rough structure indicates the molecular entanglement between the chains of PAM/PAA and PVA. This morphological change explains the swelling capacity of the gels. With the introduction of PVA, the physical crosslinking density of the hydrogel was increased by the F-T process cycles, and the molecular entanglement between PAM, PAA and PVA was strengthened, leading to a decreased hydrogel water content but increased tensile strength. In the freeze-drying process (hydrogels dehydrated by lyophilization), the PVA of the hydrogel was frozen into a crystal area and the pores of the PAM, PAA produced by lyophilization were eventually filled with the chains or the crystal area of PVA.

\section{Fourier transform infrared (FTIR) spectroscopy}

The FTIR results of the gels are shown in Fig. 9a. A broad band appearing at $3430 \mathrm{~cm}^{-1}$ in the PVA gel spectra corresponds to stretching vibrations of the hydroxyl groups. And a band appearing in the spectra of $1095 \mathrm{~cm}^{-1}$ is related to $\mathrm{C}-\mathrm{O}$ stretching. The PAM gel showed bands at $3435 \mathrm{~cm}^{-1}$ and $3200 \mathrm{~cm}^{-1}$, assigned to a stretching vibration of $\mathrm{N}-\mathrm{H}$. The bands at $1658 \mathrm{~cm}^{-1}$ $\left(\mathrm{C}=\mathrm{O}\right.$ stretching), $1622 \mathrm{~cm}^{-1}(\mathrm{~N}-\mathrm{H}$ deformation for primary amine), $1450 \mathrm{~cm}^{-1}\left(\mathrm{CH}_{2}\right.$ in-plane scissoring), $1418 \mathrm{~cm}^{-1}$ (C-N stretching for primary amide), $1317 \mathrm{~cm}^{-1}$ (C-H deformation), and $1120 \mathrm{~cm}^{-1}\left(\mathrm{NH}_{2}\right.$ inplane rocking) were also detected. The FTIR spectra of the TN gel was shown in Fig. 9a. The characteristic peaks of the PAM and PVA gel can be found compared with them.

\section{X-ray diffraction (XRD)}

The XRD results (Fig. 9b) showed a amorphous diffraction for the PAM gels with a broad peak at $2 \theta=41$ degree. And the PVA gel exhibited a typical peak that appeared at $2 \theta=20$ degree, corresponding to the mixture of (101) and (200) plane of PVA semi-crystalline in pure PVA gel. ${ }^{39,40}$ PVA crystallinity decreased with decreasing PVA content in the TN gels. And PVA semicrystalline can be formed in TN gels. The molecular entanglement between PAM, PAA and PVA could account for the decreasing of PVA crystallinity.

\section{Swelling behaviour}

Fig. 10a displays the equilibrium swelling ratio (ESR) of the hydrogels in deionized water (DI). The swelling ability of the hydrogels increased with declining PVA content. The 2-P0-C4.17 TN gels showed a maximum swelling ratio of $1015.6 \%$, 
a

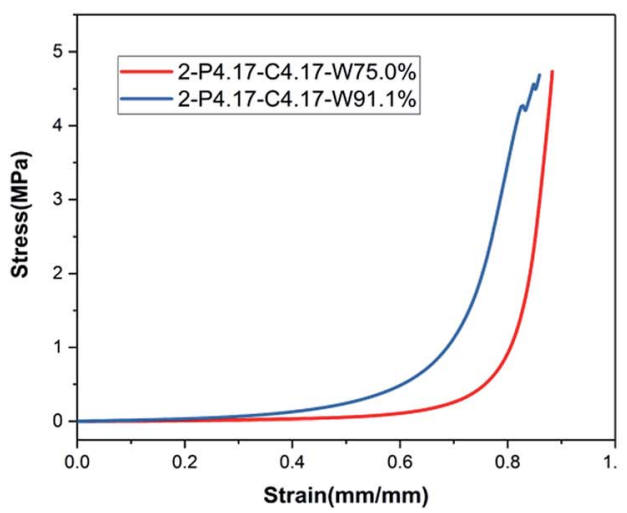

b

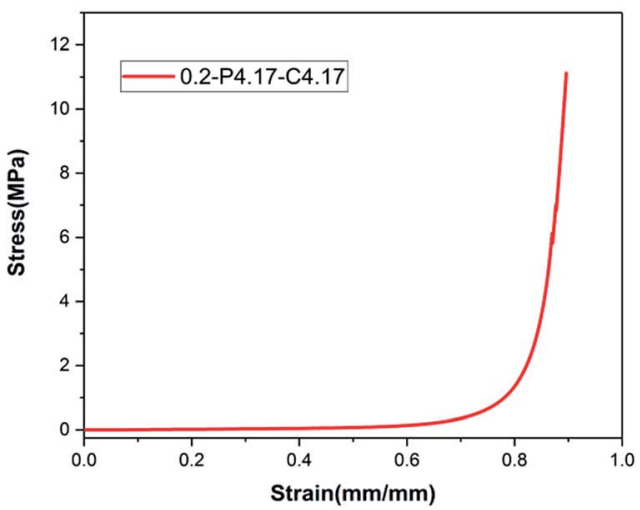

Fig. 7 (a) Compressive stress-strain curves of the 2-P4.17-C4.17 TN gels with different content of water. (b) Compressive stress-strain curves of the 0.2-P4.17-C4.17 TN gel with a total of three cycles of the F - T process.

a

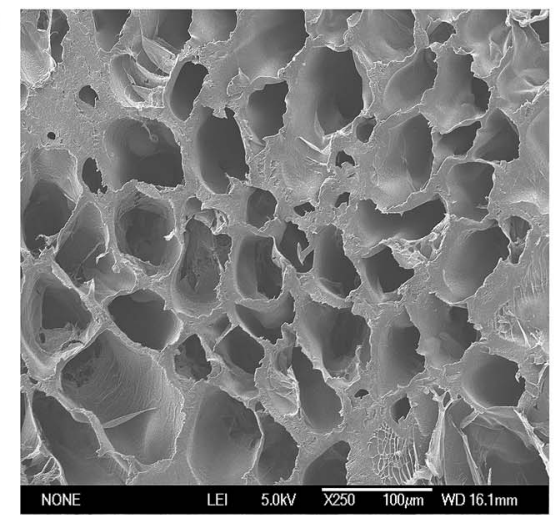

c

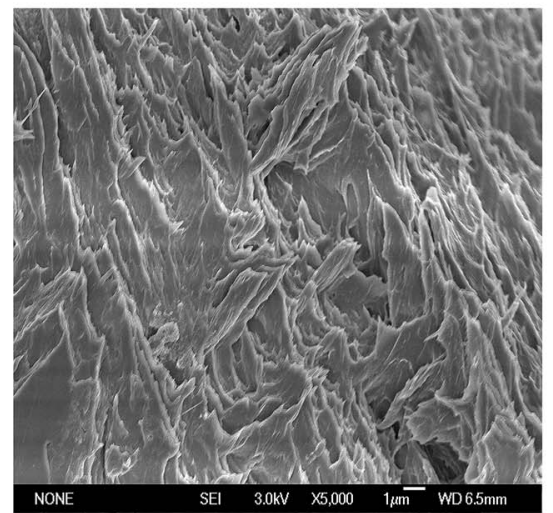

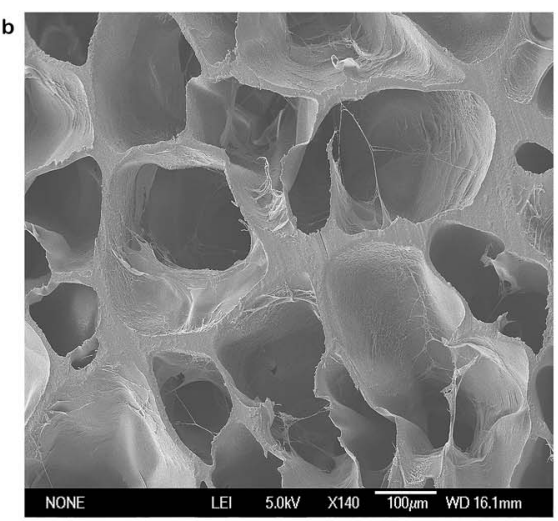

d

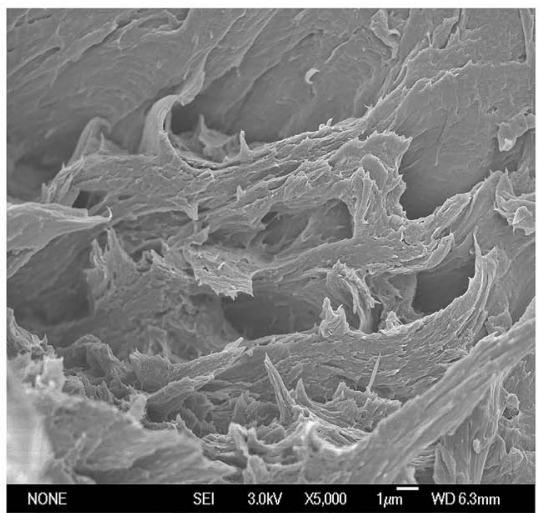

Fig. 8 (a, b) SEM images of PAM hydrogels. (c, d) SEM images of the 2-P4.17-C0.21 TN gels.

compared to $587.0 \%$ for the 2-P8.33-C4.17 TN gels. Less crosslinked hydrogels tended to show higher water absorption ability, because the highly cross-linked structure could not sustain a large amount of water within the gel structure, reducing the space and ability to take up water during the $\mathrm{F}-\mathrm{T}$ process. For the TN gels, when the PVA content decreased, the physical cross-linked density of the hydrogel network decreased, and molecular entanglement between PAM/PAA and PVA was weakened, which led to improvement in the hydrogel swelling ability. At the same time, the swelling capability was strongly influenced by the hydrophilicity of the hydrogels. The swelling ability of the gels, with a changing AM/AA ratio, is shown in Fig. 10b. By enhancing the weight ratio of AM/AA, the crosslinking between PAA and $\mathrm{Ca}^{2+}$ decreased, and the hydrophilic groups of the hydrogel networks increased, and consequently, the hydrogel absorbed more water, demonstrating improved swelling ability. Fig. 10c shows the ESR of the hydrogels in DI with changing $\mathrm{CaCl}_{2}$; the weight ratio of AM/AA and PVA were unchanged. Furthermore, the ESR of the hydrogels gradually increased with the decreasing $\mathrm{CaCl}_{2}$ content, excess $\mathrm{CaCl}_{2}$ was 
a

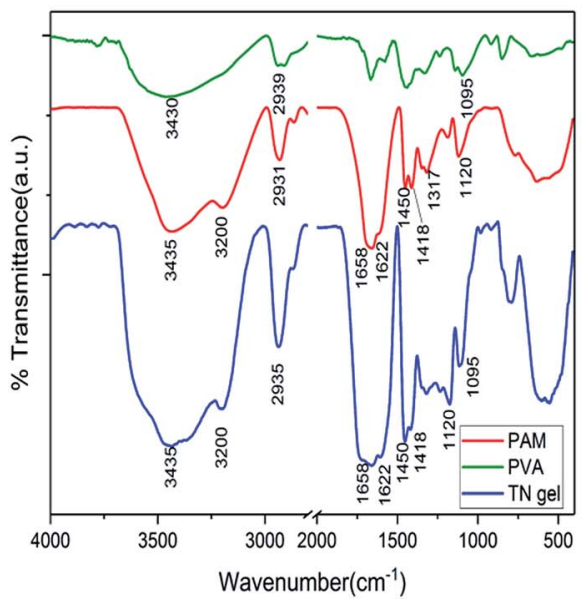

b

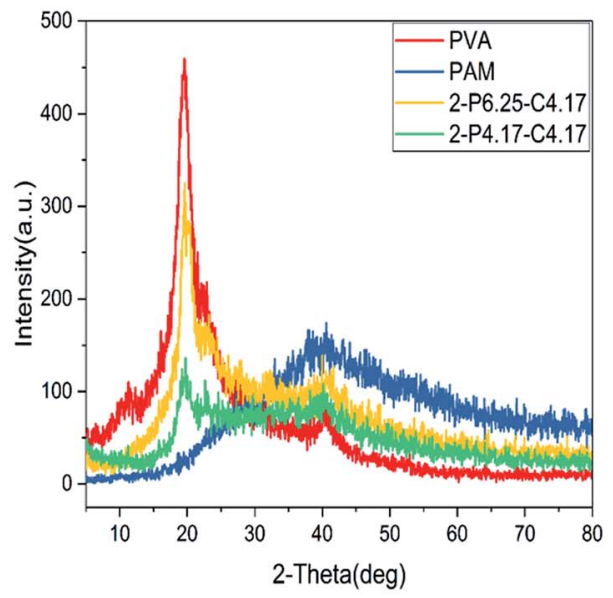

Fig. 9 (a) FTIR spectra of pure PVA, PAM and TN gel. (b) XRD patterns of PVA, PAM, 2-P6.25-C4.17 TN and 2-P4.17-C4.17 TN gels.

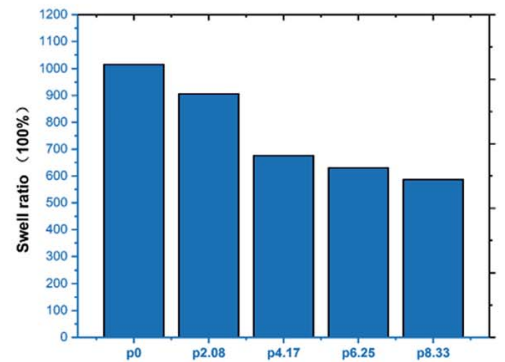

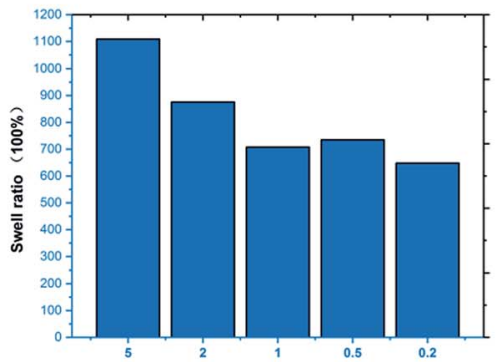

c

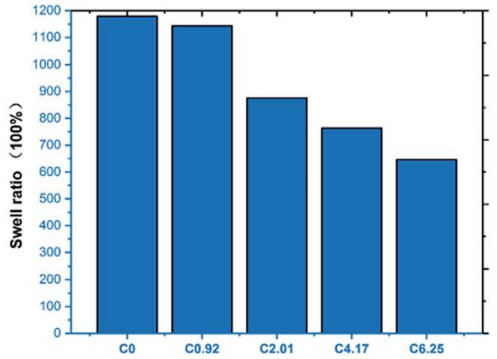

Fig. 10 (a) The swelling ability of the hydrogels at various weight ratio of AM/AC. (b) The swelling ability of the prepared hydrogels at various content of PVA. (c) The swelling ability of the prepared hydrogels at different content of $\mathrm{CaCl}_{2}$.

cross-linked with PAA with the increasing AA content. Therefore, the hydrophilic groups were decreased, leading to a decreased swelling ability. The swelling ability of the prepared hydrogels can be improved by controlling the ratio of AA/AM, AM/PVA and $\mathrm{AA} / \mathrm{CaCl}_{2}$, according to the swelling experiments.

\section{Experimental}

\section{Materials}

$\mathrm{AM}, \mathrm{AA}$, and $\mathrm{CaCl}_{2}$ were purchased from Kermel. PVA (average $M_{\mathrm{w}}$ $=89000 \mathrm{~g} \mathrm{~mol}^{-1}$ ) was purchased from Sinopharm Chemical Co, Ltd. Ammonium persulfate (APS), $N, N, N^{\prime}, N^{\prime}$-tetramethylethylenediamine (TEMED) and $N, N^{\prime}$-methylenebisacrylamide (BIS) were purchased from Energy Chemical. The concentrations of the stock solution of TEMED (liquid), APS, and BIS were fixed at $5 \% \mathrm{v} / \mathrm{v}, 1 \%$ $\mathrm{w} / \mathrm{v}$ and $1 \% \mathrm{w} / \mathrm{v}$, respectively. All materials were used as received.

\section{Preparation of TN gels}

The hydrogels were prepared using a two-step method. First, the hydrogels were synthesized through an in situ radical polymerization of the monomers (AM and AA) in an aqueous solution of PVA with a small amount of BIS, which acted as a cross-linker.
The TN gels were then obtained by an $\mathrm{F}-\mathrm{T}$ process. The initial concentration of AM plus AA was kept at $25 \% \mathrm{w} / \mathrm{v}$, whereas the concentration of PVA was varied between 0 and $8.33 \% \mathrm{w} / \mathrm{v}$; the concentrations of TEMED, APS, and BIS were $0.208 \% \mathrm{v} / \mathrm{v}$, $0.083 \% \mathrm{w} / \mathrm{v}$, and $0.025 \% \mathrm{w} / \mathrm{v}$, respectively. First, PVA was added to DI and stirred continuously at $90{ }^{\circ} \mathrm{C}$ for about $2 \mathrm{~h}$ to form a homogeneous, transparent solution with the designated concentration. AM, AA, $\mathrm{CaCl}_{2}$, BIS and PVA solutions were then added into a flask, and the solution was stirred vigorously with a magnetic stirrer at room temperature for $2 \mathrm{~h}$. Argon gas was bubbled through the solution for $30 \mathrm{~min}$, and the APS $(1 \mathrm{ml})$ and TEMED $(1 \mathrm{ml})$ were introduced. The mixture solution was transferred into the mould, and the radical polymerization was carried out in a water bath at $65^{\circ} \mathrm{C}$ for $8 \mathrm{~h}$. The hydrogels were removed from the mould after polymerization, frozen at $-20^{\circ} \mathrm{C}$ for $12 \mathrm{~h}$ and thawed at $25^{\circ} \mathrm{C}$ for $8 \mathrm{~h}$, for three consecutive cycles. Finally, the samples were stored in the as-prepared state for mechanical property testings.

In this article, the as prepared TN gels are denoted as $x-\mathrm{P} y$ $\mathrm{C} z$, where $\mathrm{P}$ and $\mathrm{C}$ indicate PVA and $\mathrm{CaCl}_{2}$, respectively, $x$ represents the weight ratio of $\mathrm{AM} / \mathrm{AA}$, and $y, z$ represent the corresponding concentrations in the form PVA/DI (w/v) and $\mathrm{CaCl}_{2} / \mathrm{DI}(\mathrm{w} / \mathrm{v})$, respectively. For example, 2-P2.1-C0.92 TN gels 
represent a gel containing AM/AA with a ratio of 2, PVA with a PVA/DI ratio of $2.1 \% \mathrm{w} / \mathrm{v}$ and $\mathrm{CaCl}_{2}$ with a $\mathrm{CaCl}_{2} / \mathrm{DI}$ ratio of $0.92 \% \mathrm{w} / \mathrm{v}$. FT represents the Freezing-thawing process. S and $\mathrm{W}$ represent the cycles of loading-unloading experiments and water content, respectively.

\section{Measurements of mechanical properties}

Tensile and compressive tests were performed on the asprepared hydrogel samples with a Universal Testing Machine (WDW-10) at room temperature. The hydrogel samples for tensile testing were rod-like shapes $50 \mathrm{~mm}$ in length, while the diameter of the as-prepared hydrogel samples was $7 \mathrm{~mm}$, with a crosshead speed of $100 \mathrm{~mm} \mathrm{~min}{ }^{-1}$. The tensile strain was taken as the length change related to the initial length, and the tensile stress was evaluated on the cross-section of the initial sample. The toughness was calculated from the area of the stress-strain curves. For compression testing, cylindrical samples of the as-prepared hydrogel were $(30 \mathrm{~mm}$ diameter and $30 \mathrm{~mm}$ height). The crosshead speed was $5 \mathrm{~mm} \mathrm{~min}^{-1}$, and the compression strength was obtained using the stress at deformation of $90 \%$. The hysteresis of the as-prepared gels samples was measured at a deformation of $80 \%$.

\section{Swelling experiments}

The swelling properties of the hydrogel were characterized by a gravimetric method. ${ }^{41}$ The dried hydrogels were immersed in excess DI to reach the state of swelling equilibrium at room temperature. The equilibrium swelling ratio was calculated as:

$$
\mathrm{ESR}=\frac{W_{\mathrm{s}}-W_{\mathrm{d}}}{W_{\mathrm{d}}}
$$

where $W_{\mathrm{s}}$ is the weight of the swollen gel and $W_{\mathrm{d}}$ is the weight of the dry gel.

\section{Scanning electron microscopy (SEM)}

The surface morphology of the hydrogels after being freeze dried was investigated by scanning electron microscope (JSM6700F, Japan) using $3.0 \mathrm{kV}$ voltage. Prior to observation, the freeze dried fracture sections were coated with a thin layer of platinum to enhance conductivity.

\section{X-ray diffraction (XRD)}

A D8 Advance X-ray diffractometer (Bruker, Germany, $40 \mathrm{kV}$, $40 \mathrm{~mA}, \mathrm{Cu}(\mathrm{K} \alpha)$ radiation, $\lambda=1.54184 \AA)$ was used to measure the crystallinity of the TN gels. The XRD results were obtained from $5^{\circ}$ to $80^{\circ}, 2 \theta$ at a rate of $10^{\circ} \mathrm{min}^{-1}$.

\section{Fourier transform infrared (FTIR) spectroscopy}

The hydrogels were freeze-dried by a lyophilizer for $24 \mathrm{~h}$ at $-50{ }^{\circ} \mathrm{C}$ to eliminate water molecules from the gels. FTIR spectra were recorded between 400 and $4000 \mathrm{~cm}^{-1}$ on a FTIR Spectrometer from Bruker Optics Inc.

\section{Conclusions}

In this work, we synthesized and characterized TN gels. A PAM, PAA network and PVA network can be simultaneously mixed and interspersed together in a unique one-pot, two-step reaction, with the resulting hydrogels having anti-fatigue and supertough properties. The PAM, PAA network provided a rigid scaffold for holding up the shape of the TN gels, whereas the PVA polymers in the PAM/PAA networks play a role in energy absorption. Some PVA chains entangled with the PAM, PAA chains, and the PAA and $\mathrm{Ca}^{2+}$ made another network by ionic cross-linking. This simple two-step method is particularly interesting when a chemical, physical and ionic network is mixed into the hydrogels to enhance the mechanical strength. Even hydrogels with high water content had the excellent compressive performance. The mechanical properties and absorbing ability of the TN gels can be adjusted by regulating the concentration of PVA and the number of F-T cycles; the compressive stress can be regulated by controlling the AM/AA weight ratio. The as-prepared hydrogels have more potential applications as materials for articular cartilage and artificial muscles.

\section{Conflicts of interest}

There are no conflicts to declare.

\section{Acknowledgements}

This work was financially supported by the National Natural Science Foundation of China (No. 21774070 and 21274080) and Fund for Shandong province major scientific and technological innovation projects (No. 2017CXGC1112).

\section{Notes and references}

1 C. Zhang, G. G. Cano and P. V. Braun, Adv. Mater., 2014, 26, 5678-5683.

2 Y. Wang, L. Wang, T. Yang, X. Li, X. Zang, M. Zhu, K. Wang, D. Wu and H. Zhu, Adv. Funct. Mater., 2014, 24, 4666-4670.

3 J. Lu, C. Cheng, Y. S. He, C. Lyu, Y. Wang, J. Yu, L. Qiu, D. Zou and D. Li, Adv. Mater., 2016, 28, 4025-4031.

4 M. K. Shin, G. M. Spinks, S. R. Shin, S. I. Kim and S. J. Kim, Adv. Mater., 2009, 21, 1712-1715.

5 B. V. Slaughter, S. S. Khurshid, O. Z. Fisher, A. Khademhosseini and N. A. Peppas, Adv. Mater., 2009, 21, 3307-3329.

6 N. Annabi, S. R. Shin, A. Tamayol, M. Miscuglio, M. A. Bakooshli, A. Assmann, P. Mostafalu, J. Y. Sun, S. Mithieux, L. Cheung, X. S. Tang, A. S. Weiss and A. Khademhosseini, Adv. Mater., 2016, 28, 40-49.

7 M. A. Haque, T. Kurokawa and J. P. Gong, Polymer, 2012, 53, 1805-1822.

8 K. Yasuda, N. Kitamura, J. P. Gong, K. Arakaki, H. J. Kwon, S. Onodera, Y. M. Chen, T. Kurokawa, F. Kanaya, Y. Ohmiya and Y. Osada, Macromol. Biosci., 2009, 9, 307-316. 
9 K. Haraguchi and T. Takada, Macromolecules, 2010, 43, 42944299.

10 E. Ozturk, O. Arlov, S. Aksel, L. Li, D. M. Ornitz, G. SkjakBraek and M. Zenobi-Wong, Adv. Funct. Mater., 2016, 26, 3649-3662.

11 J. Liu, D. Su, J. Yao, Y. Huang, Z. Shao and X. Chen, J. Mater. Chem. A, 2017, 5, 4163-4171.

12 E. Tous, J. L. Ifkovits, K. J. Koomalsingh, T. Shuto, T. Soeda, N. Kondo, J. H. Gorman III, R. C. Gorman and J. A. Burdick, Biomacromolecules, 2011, 12, 4127-4135.

13 T. L. Sun, T. Kurokawa, S. Kuroda, A. B. Ihsan, T. Akasaki, K. Sato, M. A. Haque, T. Nakajima and J. P. Gong, Nat. Mater., 2013, 12, 932-937.

14 S. Hong, D. Sycks, H. F. Chan, S. Lin, G. P. Lopez, F. Guilak, K. W. Leong and X. Zhao, Adv. Mater., 2015, 27, 4035-4040.

15 W. Sun, B. Xue, Y. Li, M. Qin, J. Wu, K. Lu, J. Wu, Y. Cao, Q. Jiang and W. Wang, Adv. Funct. Mater., 2016, 26, 90449052.

16 X. Qin, F. Zhao, Y. Liu, H. Wang and S. Feng, Colloid Polym. Sci., 2009, 287, 621-625.

17 M. Burnworth, L. Tang, J. R. Kumpfer, A. J. Duncan, F. L. Beyer, G. L. Fiore, S. J. Rowan and C. Weder, Nature, 2011, 472, 334-337.

18 P. Lin, S. Ma, X. Wang and F. Zhou, Adv. Mater., 2015, 27, 2054-2059.

19 M. X. Wang, C. H. Yang, Z. Q. Liu, J. Zhou, F. Xu, Z. Suo, J. H. Yang and Y. M. Chen, Macromol. Rapid Commun., 2015, 36, 465-471.

20 L. Zhang, N. R. Brostowitz, K. A. Cavicchi and R. A. Weiss, Macromol. React. Eng., 2014, 8, 81-99.

21 Z. Wei, J. He, T. Liang, H. Oh, J. Athas, Z. Tong, C. Wang and Z. Nie, Polym. Chem., 2013, 4, 4601.

22 Z. Li, Z. Hou, H. Fan and H. Li, Adv. Funct. Mater., 2017, 27, 1604379.

23 J. Hou, X. Ren, S. Guan, L. Duan, G. H. Gao, Y. Kuai and H. Zhang, Soft Matter, 2017, 13, 1357-1363.
24 Q. Chen, L. Zhu, C. Zhao, Q. Wang and J. Zheng, Adv. Mater., 2013, 25, 4171-4176.

25 H. M. Nizam El-Din, S. G. Abd Alla and A. W. M. El-Naggar, J. Macromol. Sci., Part A: Pure Appl. Chem., 2007, 44, 47-54.

26 J. P. Gong, Soft Matter, 2010, 6, 2583.

27 R. E. Webber, C. Creton, H. Brown and J. P. Gong, Macromolecules, 2007, 40, 2919-2927.

28 J. Y. Sun, X. Zhao, W. R. Illeperuma, O. Chaudhuri, K. H. Oh, D. J. Mooney, J. J. Vlassak and Z. Suo, Nature, 2012, 489, 133136.

29 T. R. Hoare and D. S. Kohane, Polymer, 2008, 49, 1993-2007. 30 H. Li, D. Hao, J. Fan, S. Song, X. Guo, W. Song, M. Liu and L. Jiang, J. Mater. Chem. B, 2016, 4, 4662-4666.

$31 \mathrm{H}$. Bodugoz-Senturk, C. E. Macias, J. H. Kung and O. K. Muratoglu, Biomaterials, 2009, 30, 589-596.

32 Q. Wei, Y. Wang, Y. Che, M. Yang, X. Li and Y. Zhang, J. Mech. Behav. Biomed. Mater., 2017, 65, 565-573.

33 L. Zhang, J. Zhao, J. Zhu, C. He and H. Wang, Soft Matter, 2012, 8, 10439.

34 M. Fukasawa, T. Sakai, U.-i. Chung and K. Haraguchi, Macromolecules, 2010, 43, 4370-4378.

35 L. W. Xia, R. Xie, X. J. Ju, W. Wang, Q. Chen and L. Y. Chu, Nat. Commun., 2013, 4, 2226.

36 X. Zhao, Soft Matter, 2014, 10, 672-687.

37 J. Wang, L. Lin, Q. Cheng and L. Jiang, Angew. Chem., Int. Ed., 2012, 51, 4676-4680.

38 Z. Hu and G. Chen, Adv. Mater., 2014, 26, 5950-5956.

39 S. S. Kulkarni, A. A. Kittur, M. I. Aralaguppi and M. Y. Kariduraganavar, J. Appl. Polym. Sci., 2004, 94, 13041315.

40 L. Lu, F. Peng, Z. Jiang and J. Wang, J. Appl. Polym. Sci., 2006, 101, 167-173.

41 F. Zhao, X. Qin and S. Feng, RSC Adv., 2016, 6, 100511100518. 